\title{
Development of severe bronchopulmonary dysplasia is associated with alterations in fecal volatile organic compounds
}

\author{
Daniel J.C. Berkhout ${ }^{1}$, Hendrik J. Niemarkt ${ }^{2}$, Marc A. Benninga ${ }^{1}$, Andries E. Budding ${ }^{3}$, Anton H. van Kaam ${ }^{4}$, Boris W. Kramer ${ }^{5}$, \\ Charlene M. Pantophlet ${ }^{6}$, Mirjam M. van Weissenbruch ${ }^{7}$, Nanne K.H. de Boer ${ }^{8}$ and Tim G.J. de Meij ${ }^{6}$
}

BACKGROUND: The aim of this study was to evaluate the potential of fecal volatile organic compounds (VOCs), obtained by means of an electronic nose device (Cyranose 320), as early non-invasive biomarker for BPD.

METHODS: In this nested case-control study performed at three Neonatal Intensive Care Units, fecal samples obtained at postnatal age of $7,14,21$, and 28 days from preterm infants with severe bronchopulmonary dysplasia (BPD) were compared with fecal VOC profiles from matched controls. Microbiota analysis was performed by means of IS-pro technique on fecal samples collected at 28 days postnatally.

RESULTS: VOC profiles of infants developing severe BPD $(n=15)$ could be discriminated from matched controls $(n=15)$ at postnatal age of 14 days (area under the curve $( \pm 95 \%$ confidence interval), $P$-value, sensitivity, specificity; 0.72 (0.54$0.90), 0.040,60.0 \%, 73.3 \%), 21$ days (0.71 (0.52-0.90), 0.049, $66.7 \%, 73.3 \%)$ and 28 days (0.77 (0.59-0.96), 0.017, 69.2\%, 69.2\%) but not at 7 days. Intestinal microbiota did not differ between BPD subjects and controls.

CONCLUSION: Fecal VOC profiles of infants developing BPD could be differentiated from controls at postnatal day 14, 21, and 28. VOC differences could not be directed to intestinal microbiota alterations but presumably reflect local and systemic metabolic and inflammatory pathways associated with BPD.

B ronchopulmonary dysplasia (BPD) is the most common adverse outcome in preterm, very low birth weight infants $(<1500 \mathrm{~g})$, with an overall incidence of approximately $30 \%$ (1). BPD is associated with significant morbidity and mortality, prolonged hospital stay, and increased financial burden (2). Furthermore, survivors of BPD commonly suffer from impaired cognitive functions (3) and frequently demonstrate prolonged lung function abnormalities (4-6). Interventions that reduce the incidence of BPD are therefore urgently needed. However, to date, most intervention studies failed to show a clear reduction in BPD or this reduction was accompanied by serious adverse effects (7). Possible explanations include the poor ability to predict the risk of BPD at an early stage, thereby failing to identify those patients who will benefit most from interventions aimed at reducing the risk for BPD. Identification of novel, preferably non-invasive biomarkers for early prediction of BPD remains a priority.

Volatile organic compounds (VOCs) are carbon-based, odorous chemicals produced during both physiological and pathophysiological processes and can be obtained noninvasively from all conceivable bodily excrements (e.g., feces, sweat, exhaled air, urine, etc.) (8). Over the past years, a rapidly increasing number of studies have investigated the potential of VOCs as diagnostic biomarker for several metabolic (9), malignant (10), infectious (11), and inflammatory diseases (12). As BPD is a pulmonary disease, exhaled breath or tracheal aspirates would ideally be the substrate of choice for VOC analysis. However, as majority of preterm infants receive non-invasive respiratory support, collection of these samples is complex or not feasible. Fecal VOCs are an interesting alternative for exhaled VOCs, especially because animal and adult human studies have demonstrated the existence of vital cross-talks between different mucosal sites of the body, including a bidirectional interaction between gut and lungs, the so-called gut-lung axis $(13,14)$.

Consequently, fecal VOCs might be a promising candidate for identifying preterm infants at risk of developing BPD. Therefore, the aim of the current study was to assess whether infants developing severe BPD could be discriminated from controls by fecal VOC profiles at different consecutive postnatal time points. Additional microbiota analysis was performed to evaluate whether potential differences in VOC profiles were mediated by changes in intestinal microbiome.

\section{METHODS}

\section{Subjects}

This nested case-control study was conducted between October 2014 and June 2016 at three Neonatal Intensive Care Units in the

\footnotetext{
'Department of Pediatric Gastroenterology, Emma Children's Hospital/Academic Medical Center, Amsterdam, The Netherlands; ${ }^{2}$ Neonatal Intensive Care Unit, Máxima Medical Center, Veldhoven, The Netherlands; ${ }^{3}$ Department of Microbiology, VU University Medical Center, Amsterdam, The Netherlands; ${ }^{4}$ Neonatal Intensive Care Unit, Emma Children's Hospital/Academic Medical Center, Amsterdam, The Netherlands; ${ }^{5}$ Department of Pediatrics, Maastricht University Medical Center, Maastricht, The Netherlands; ${ }^{6}$ Department of Pediatric Gastroenterology, VU University Medical Center, Amsterdam, The Netherlands; ${ }^{7}$ Neonatal Intensive Care Unit, VU University Medical Center, Amsterdam, The Netherlands; ${ }^{8}$ Department of Gastroenterology and Hepatology, VU University Medical Center, Amsterdam, The Netherlands. Correspondence: Daniel J.C. Berkhout (d.berkhout@vumc.nl) 
Netherlands: VU medical center, Academic Medical Center (Amsterdam), and Máxima Medical Center (Veldhoven). This study was approved by the local institutional review boards of all participating centers (A2016.225). Parental informed consent was obtained from all included infants. None of the participating medical centers administered probiotics.

Infants born at a gestational age of $\leqslant 30$ weeks were eligible to participate in this study. Exclusion criteria included congenital gastrointestinal anomalies (e.g., anal atresia, Hirschsprung's disease), intestinal surgery, necrotizing enterocolitis (any stage), and pneumonia (defined as clinical symptoms of respiratory infection and initiation of adequate antibiotic treatment based on the pathogen isolated from the tracheal aspirate culture) during the first 28 days of life. Infants diagnosed with severe BPD, defined as supplemental oxygen dependency for at least 28 days of life and requiring positive pressure support and/or receiving $>30 \%$ oxygen at 36 weeks postmenstrual age (PMA) (15) were allocated to the BPD group. The control group was selected from the remaining infants after exclusion of infants with mild or moderate BPD (15). In addition to standard demographic data, information about mechanical ventilation, supplementary oxygen requirement, postnatal infection, sepsis, medication use, and feeding pattern was prospectively collected for each included infant. If an infant was transferred to another hospital before reaching the PMA of 36 weeks, information on the presence and severity of BPD was obtained from medical records of the hospitals to which these infants were transferred.

\section{Fecal Sample Collection}

Fecal samples were collected daily from the diaper by the nurse until the infant reached the postnatal age of 28 days. Fecal samples were collected in a stool container (Stuhlgefäß $10 \mathrm{ml}$, Frickenhausen, Germany) and stored at a temperature of $-20^{\circ} \mathrm{C}$ within $15 \mathrm{~min}$ after collection. If daily bowel movements were absent, the subsequently produced fecal sample was collected. If the infant deceased, got discharged, or transferred to another hospital before reaching the age of 28 days, stool sampling was prematurely terminated. All samples were transported in frozen condition to the VU medical center and stored at $-20^{\circ} \mathrm{C}$ until further handling.

\section{Sample Selection}

Fecal samples collected at a postnatal age of 7, 14, 21, and 28 days were selected for further VOC analysis. Each fecal sample from an infant diagnosed with severe BPD at 36 weeks PMA was strictly matched to one fecal sample of a control subject. Matching was based on: center of birth, postnatal age, gestational age, birth weight, enteral feeding pattern (breastmilk, formula, or combination of both), and cumulative number of days exposed to antibiotics. In case a fecal sample of an infant with BPD was absent at a given time point, an available sample collected within 2 days adjacent to the missing sample was selected for analysis. If no sample was available, both case and control were excluded at this given time point.

\section{Fecal VOC Analysis by Electronic Nose}

Fecal VOC analysis was performed using an electronic nose device (Cyranose 320, Smiths detection, Pasadena, CA). Sampling and measurement conditions were in accordance with the methods described in previous studies on fecal VOC analysis conducted by our research group $(10,12,16-18)$. In summary, approximately $0.2 \mathrm{~g}$ feces was transferred into a $3 \mathrm{ml}$ sealed vacutainer (BD vacutainer, Belliver Industrial Estate, Plymouth, UK) and gradually heated to room temperature for $30 \mathrm{~min}$, allowing fecal VOCs to fill the headspace. Subsequently, two needles (BD blunt fill needle $1.2 \times 40 \mathrm{~mm}^{2}$, Becton Dickinson, Franklin Lakes, NJ) were pierced into the cap of the vacutainer and connected to the eNose by an oxygen tube (Argyle Covidien tube $3 \mathrm{~mm}$, Argyle Covidien, Mansfield, MA), establishing a closed loop and thereby preventing dilution with ambient air. After the baseline reference was obtained by connecting a VOC filter (A1, North Safety, Middelburg, The Netherlands), the actual VOC analysis was performed by letting fecal
VOCs pass the array of 32 sensors for $60 \mathrm{~s}$. Each sensor consists of a unique nonconductive polymer coating containing conductive carbon black material particles (19). VOC presentation leads to swelling of the polymer coating, resulting in an increase in electrical resistance due to augmentation of the distance between the conductive particles (19). Each sensor coating being unique, eventually leads to 32 unique electrical resistance changes forming a vapor-specific 'smell-print', comparable based on pattern recognition algorithms (19). After each measurement, sensors were purged for $90 \mathrm{~s}$ to remove any remaining fecal VOCs. All samples were analyzed in random order, and during the eNose measurements, the investigators were blinded to clinical data.

\section{Microbiota Analysis}

Fecal samples collected at postnatal age of 28 days were prepared and analyzed by means of standard IS-pro procedure, a standardized eubacterial molecular detection technique to characterize the microbiota (20). IS-pro makes use of phylum-specific fluorescently labeled PCR primers and differentiates bacterial species by the length of the 16S-23S rDNA IS region. A detailed description of the used protocol on DNA isolation and sample preparation has been previously reported $(20,21)$. In short, the procedure consists of two separate multiplex PCRs: the first PCR contains two different fluorescently labeled primers. One amplifying the phyla Firmicutes, Actinobacteria, Fusobacteria, and Verrucomicrobia (FAFV) and the other labeled primer for the phylum Bacteroidetes. A separate PCR with a third labeled primer is performed for the phylum Proteobacteria. The resulting labeled fragments were analyzed on an ABI Prism 3500 Genetic Analyzer (Applied Biosystems, Carlsbad, CA).

\section{Data Analysis: Electronic Nose}

Data analysis was performed using the Statistical Package for the Social Science (SPSS) software version 22 (SPSS, Chicago, IL). Basic demographic and clinical data were compared with a non-parametric test, a chi-square test, or independent $t$-test where appropriate. Normal distribution was checked for each individual sensor. Principal component analysis captured the raw sensor variance of the original data set containing data from all 32 sensors into four principal components. An independent $t$-test was used to assess discriminating principal components with respect to the study groups. A $P$-value of $<0.05$ was defined as being statistically significant. Discriminative principal components were used to construct a receiver operator characteristic curve with associated 95\% confidence interval and $P$-value. Overall accuracy and associated sensitivity, specificity, positive likelihood ratios, negative likelihood ratios, positive predictive values, and negative predictive values were obtained after internal cross-validation, by means of leave-one out method.

\section{Data Analysis: IS-pro}

IS-pro data were preprocessed and further analyzed with the Spotfire (TIBCO, Palo Alto, CA) software package. Similarity between profiles was calculated with cosine distance on log2-transformed data. Fragments were assigned a taxonomic classification based on fragment length and fluorescent label. Variability in microbiota composition was depicted as principal coordinate analysis, based on cosine distance measures. Within-sample microbial diversity was calculated as the Shannon diversity index with the R 2.15 .2 software package. Diversity was calculated both per phylum and for overall microbial composition (by pooling the phyla FAFV, Bacteroidetes and Proteobacteria together).

\section{RESULTS}

\section{Patient Population}

In total, fecal samples were collected from 286 infants and consequently were eligible to participate in this study. Based on the exclusion criteria, 76 (26.6\%) infants were excluded (Figure 1). Of the remaining 210 infants, data on the presence and severity of BPD was collected from medical records from 


\section{Articles $\mid$ Berkhout et al.}

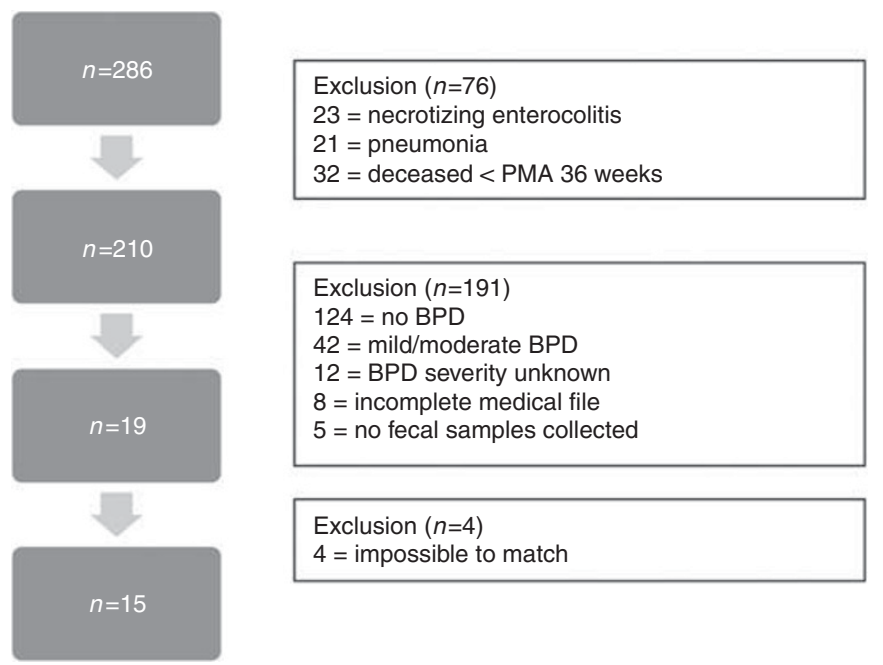

Figure 1. Flow chart of participants in the study. BPD, bronchopulmonary dysplasia; PMA, postmenstrual age. A full color version of this figure is available at the Pediatric Research journal online.

29 different hospitals in the Netherlands, to which these infants were transferred after discharge from the participating Neonatal Intensive Care Units. Sixty-one (29\%) of the infants were diagnosed with BPD, of which 19 had severe BPD. Based on the strict matching criteria, 15 subjects with severe BPD were selected for further fecal VOC analysis, together providing 108 fecal samples. Twenty-five cases were excluded from further study participation based on the inability to determine BPD severity $(n=12)$, an incomplete or missing medical file $(n=8)$, or insufficient amount of feces available for further analysis $(n=5)$. The $124(59 \%)$ remaining infants met the criteria for controls, and 15 of them were selected as strictly matched controls. Table 1 provides an overview of patient characteristics of the two subgroups.

\section{Fecal VOC Analysis}

All individual sensor results were normally distributed. Based on their fecal VOC profile, patients with severe BPD could be discriminated from strictly matched controls at a postnatal age of 14, 21, and 28 days, with an accuracy ranging between $66.7 \%$ and $70.0 \%$, but not on day 7 (Table 2). An overview of the principal component analysis with corresponding $P$ values per predefined time point is depicted in Supplementary Table S1 online. Receiver operator characteristic curves (Figure 2) and scatterplots for the discrimination between samples from infants developing BPD and controls were created for each predefined time point (Supplementary Figure S1).

\section{Microbiota analysis}

Diversity analysis showed no differences between BPD and controls in Shannon diversity indices for all phyla together and per phylum (Figure 3). A heatmap consisting of microbial data from all BPD patients and controls
Table 1. Patient characteristics of cases and matched controls

\begin{tabular}{|c|c|c|}
\hline Number $(n)$ & BPD (15) & Controls (15) \\
\hline \multicolumn{3}{|l|}{ Sex } \\
\hline $\operatorname{Male}^{\dagger}(n(\%))$ & $10(67)$ & $9(60)$ \\
\hline Birth weight $^{\dagger}$ (median (IQR)), g & $870(227.5)$ & $940(202.5)$ \\
\hline $\begin{array}{l}\text { Gestational age }{ }^{\dagger} \text { (median (IQR)), weeks } \\
\text { +days (days) }\end{array}$ & $26+2(17.5)$ & $27+0(10)$ \\
\hline \multicolumn{3}{|l|}{ Way of delivery $(n=(\%))$} \\
\hline Vaginal delivery $^{\dagger}$ & $5(33)$ & $5(33)$ \\
\hline Surfactant use ${ }^{\dagger}(n(\%))$ & $11(73)$ & $7(47)$ \\
\hline $\begin{array}{l}\text { Mechanical ventilation during first } \\
28 \text { days }(n(\%)) \ddagger\end{array}$ & $11(73)$ & $5(33)$ \\
\hline $\begin{array}{l}\text { Age at the start of mechanical } \\
\text { ventilation }^{\dagger}(\text { median (IQR)), days }\end{array}$ & $1(10.3)$ & $3(4.8)$ \\
\hline $\begin{array}{l}\text { Maximum oxygen during first } 28 \text { days }^{\dagger} \\
\text { (median (IQR)), \% }\end{array}$ & $75(80)$ & $27.5(30.8)$ \\
\hline \multicolumn{3}{|l|}{ Cumulative antibiotic use (median (IQR)), days } \\
\hline Week $1^{\dagger}$ & $5(4)$ & $5(3.5)$ \\
\hline Week $2^{\dagger}$ & $10(5.5)$ & $9(6)$ \\
\hline Week $3^{\dagger}$ & $14(8.5)$ & $10(6)$ \\
\hline Week $4^{\dagger}$ & $15(10)$ & $10(8.5)$ \\
\hline \multicolumn{3}{|l|}{ Feeding pattern ${ }^{\dagger}(n(\%))$} \\
\hline Breast milk \pm formula & $14(93)$ & $14(93)$ \\
\hline Exclusive formula & $1(7)$ & $1(7)$ \\
\hline
\end{tabular}


Table 2. Performance characteristics for the discrimination of severe BPD and controls by fecal volatile organic compound analysis

\begin{tabular}{|c|c|c|c|c|c|c|c|c|c|c|}
\hline Age (days) & BPD samples ${ }^{\mathrm{a}}(n)$ & AUC $( \pm 95 \% C l)$ & $P$-value & Accuracy, \% & Sensitivity, \% & Specificity, \% & $+P V$ & $-P V$ & $+\mathrm{LR}$ & $-L R$ \\
\hline 7 & 11 & $0.58(0.33-0.83)$ & 0.533 & 50.0 & 45.5 & 54.5 & 50.0 & 50.0 & 1.0 & 1.0 \\
\hline 14 & 15 & $0.72(0.54-0.90)$ & 0.040 & 66.7 & 60 & 73.3 & 69.2 & 64.7 & 2.25 & 0.55 \\
\hline 28 & 13 & $0.78(0.59-0.96)$ & 0.017 & 69.2 & 69.2 & 69.2 & 69.2 & 69.2 & 2.25 & 0.45 \\
\hline
\end{tabular}

AUC $\pm 95 \% \mathrm{Cl}$, area under the curve with 95\% confidence interval; BPD, bronchopulmonary dysplasia; $+\mathrm{LR}$, positive likelihood ratio; $-L R$, negative likelihood ratio;+PV, positive predictive value; - PV, negative predictive value.

${ }^{a}$ Corresponding number of fecal samples from controls were analyzed.
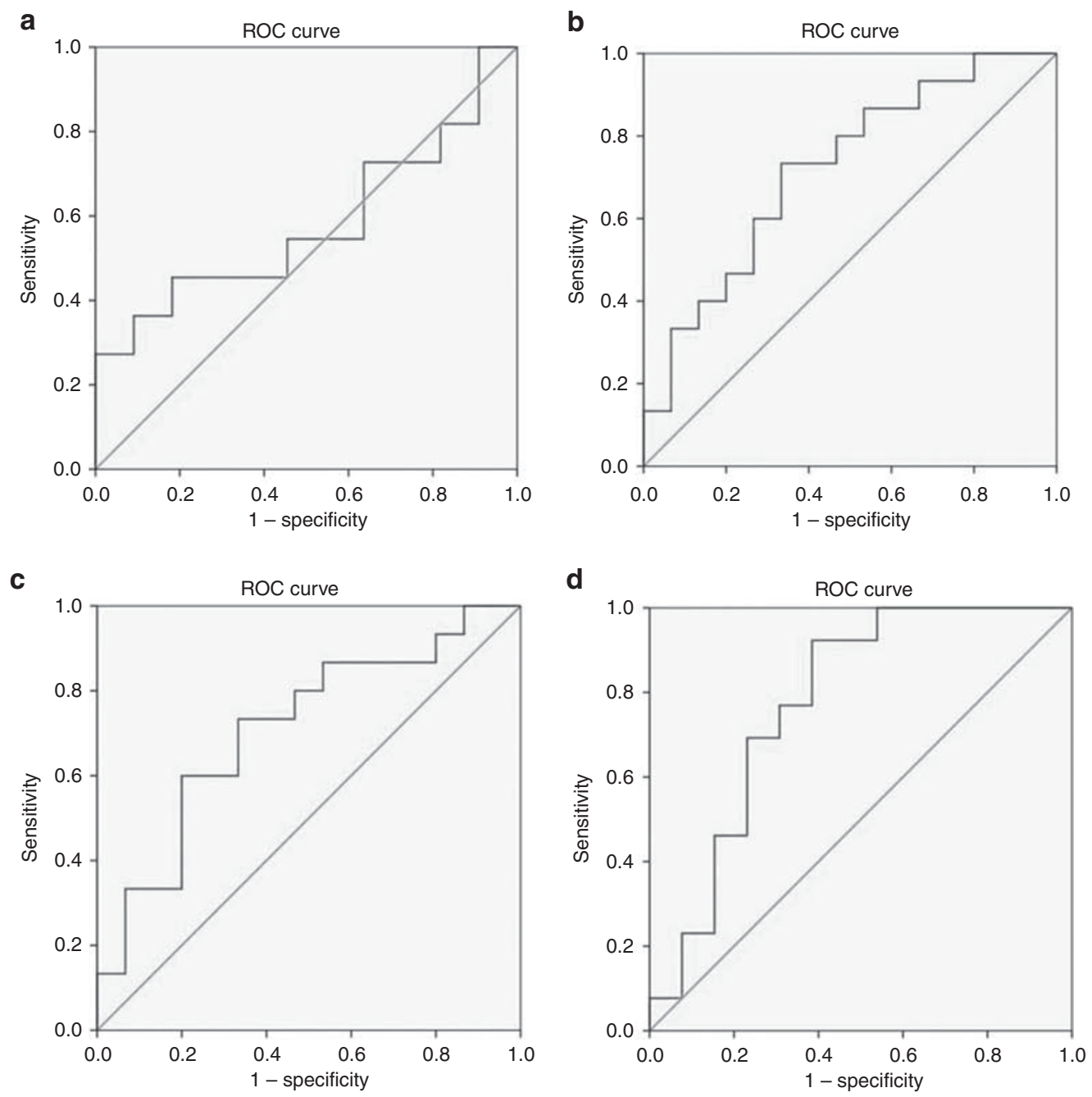

Figure 2. Receiver operator characteristic (ROC) curves for the discrimination of severe bronchopulmonary dysplasia (BPD) and controls based on fecal volatile organic compounds at the postnatal age of (a) 7 (b) $14,(\mathbf{c}) 21$, and (d) 28 days. ROC curves are constructed after internal crossvalidation. A full color version of this figure is available at the Pediatric Research journal online.

(Figure 4) and principal coordinate analysis analysis (Figure 5) revealed no disease-specific clustering, indicating that the groups could not be discriminated based on IS profiles using these unsupervised method.

\section{DISCUSSION}

In this case-control study, we found that measured fecal VOC profiles appeared to be discriminating for severe BPD compared with matched controls at postnatal age 14, 21, and 28 days but not at postnatal day 7. Intestinal microbiota composition at day 28 did not differ between severe BPD patients and controls.

There is currently only one study that assessed VOCs as potential biomarker in preterm infants at risk of developing BPD (22). In this study, VOCs deriving from tracheal secretions were measured between day 1 and day 8 after birth in preterm infants with $(n=12)$ and without $(n=11)$ $\mathrm{BPD}$, using an eNose similar to the device used in the present 


\section{Articles $\mid$ Berkhout et al.}

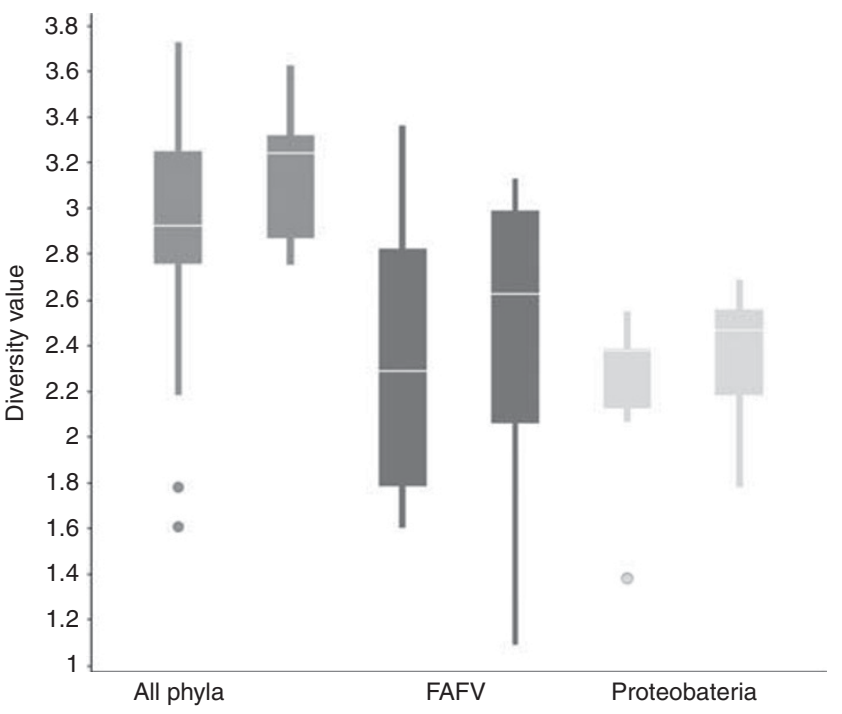

Figure 3. Microbiota diversity indices of preterm infants with severe bronchopulmonary dysplasia (BPD) and controls. Shannon diversity indices showing no differences between BPD and controls for all phyla together and per phylum. Green, all phyla together; blue, Firmicutes, Actinibacteria, Fusibacteria, and Verrucomicrobia (FAFV); yellow, Proteobacteria. Notably, Bacteriodetes are absent in all the analyzed samples. A full color version of this figure is available at the Pediatric Research journal online.

study (22). This study showed a difference in VOC profile between BPD infants and controls. However, differences in gestational age and postnatal age at sampling between the two study groups disallowed for drawing firm conclusions (22). Our study is the first to measure fecal VOCs in a wellmatched cohort study and demonstrated similar results. Our finding strengthens the hypothesis that VOC analysis has the potential to improve BPD prediction. In addition, it demonstrates that feces is a possible sample site for VOC analysis for BPD, suggesting that the gut-lung axis is involved in the pathogenesis. Notably, VOC composition was not different at day 7 postnatally. We can only speculate on explanations for this finding. First, sampling at this time point might be too early in the developmental process of BPD to detect VOC alterations that allow for discrimination between the two study groups. Second, previous studies suggest that VOCs originating from one part of the body may be transported by blood circulation before being dissolved at other bodily sites (23). As respiratory microbial community alterations in BPD infants become more apparent in time, with increasing Firmicutes and decreasing Proteobacteria (24) compared with non-BPD infants, detectable differences in fecal VOCs deriving from the respiratory microbiota may simply not be present at a postnatal age of 7 days. Moreover, as all included infants, both cases and controls, received antibiotics directly after birth for a minimum of 3 days, this may have influenced pulmonary microbiota composition, causing fecal VOC profiles originating from respiratory microbiota to be non-discriminative at postnatal age of 7 days.
However, the exact origin of the detected fecal volatiles in $\mathrm{BPD}$ infants remains yet to be elucidated (25). In addition to the volatiles deriving from the respiratory microbiota, detected alterations in fecal VOCs might also be the result of the systemic inflammatory state characterizing BPD development $(26,27)$. As VOCs may be originating from other body parts, detected differences in fecal VOCs in the current study may reflect metabolic and (patho)physiological bodily processes. Another explanation could be that BPD results in an altered respiratory microbial composition $(24,28,29)$ that changes the intestinal microbiome and vice versa by means of the gut-lung axis. For example, studies have shown that aspiration of microorganism from the gastrointestinal tract can induce microbiota alterations in the lungs (13). In addition, bacterial components and metabolites from the gut have the capacity of affecting pulmonary microbiota composition by modulation of local and systemic immunological pathways (30-32). To explore this explanation, we analyzed the intestinal microbiome of all infants at day 28 of life. Despite the differences in VOCs at this time point, the intestinal microbiome did not differ between the two study groups. Therefore, we hypothesize that the detected fecal VOC alterations resulted from local and systemic production of (volatile) biomarkers of inflammation $(33,34)$ and oxidative stress (35) related to BPD development, leading to secondary changes in fecal VOC profiles without affecting microbiota composition. The presence of elevated levels of urine clusterin, a protein indicative for oxidative stress, found in infants developing BPD in the first days of their life (36), underline this altered metabolic state. In addition to clusterin, multiple other biomarkers obtained from a wide variety of biological fluids have been proposed, representing mainly molecules associated with immunity, inflammation, and oxidative stress (37).

Findings of the current study suggest that infants developing BPD may have a distinct metabolic profile and VOC profiling may have potential as an early diagnostic biomarker for BPD development. Early prediction of BPD is needed to initiate preventive strategies in a selected population of highrisk infants and to avoid potential hazardous treatments in those not at risk for $\mathrm{BPD}$ development $(7,38,39)$. Although VOC analyses showed a fair discriminative power for severe $\mathrm{BPD}$, this finding needs to be confirmed or refuted in a larger and more heterogeneous population.

It is important to acknowledge that the eNose nose device used in this study was not specifically designed for BPD. An eNose device does not allow for identification of individual volatiles but contains an array of different gas sensors allowing for the discrimination of VOC combinations by deploying pattern recognition algorithms. Consequently, potential confounding and non-relevant VOCs may predominately interact with the eNose sensors instead of the volatiles of interest, hampering better classification (8). Future studies using chemical analytical techniques, such as gas chromatography-mass spectrometry, may allow for identification of BPD-specific VOCs. Simultaneous analysis of fecal and 


\section{Fecal volatile organic compounds in BPD $\mid$ Articles}

BPD

Controls
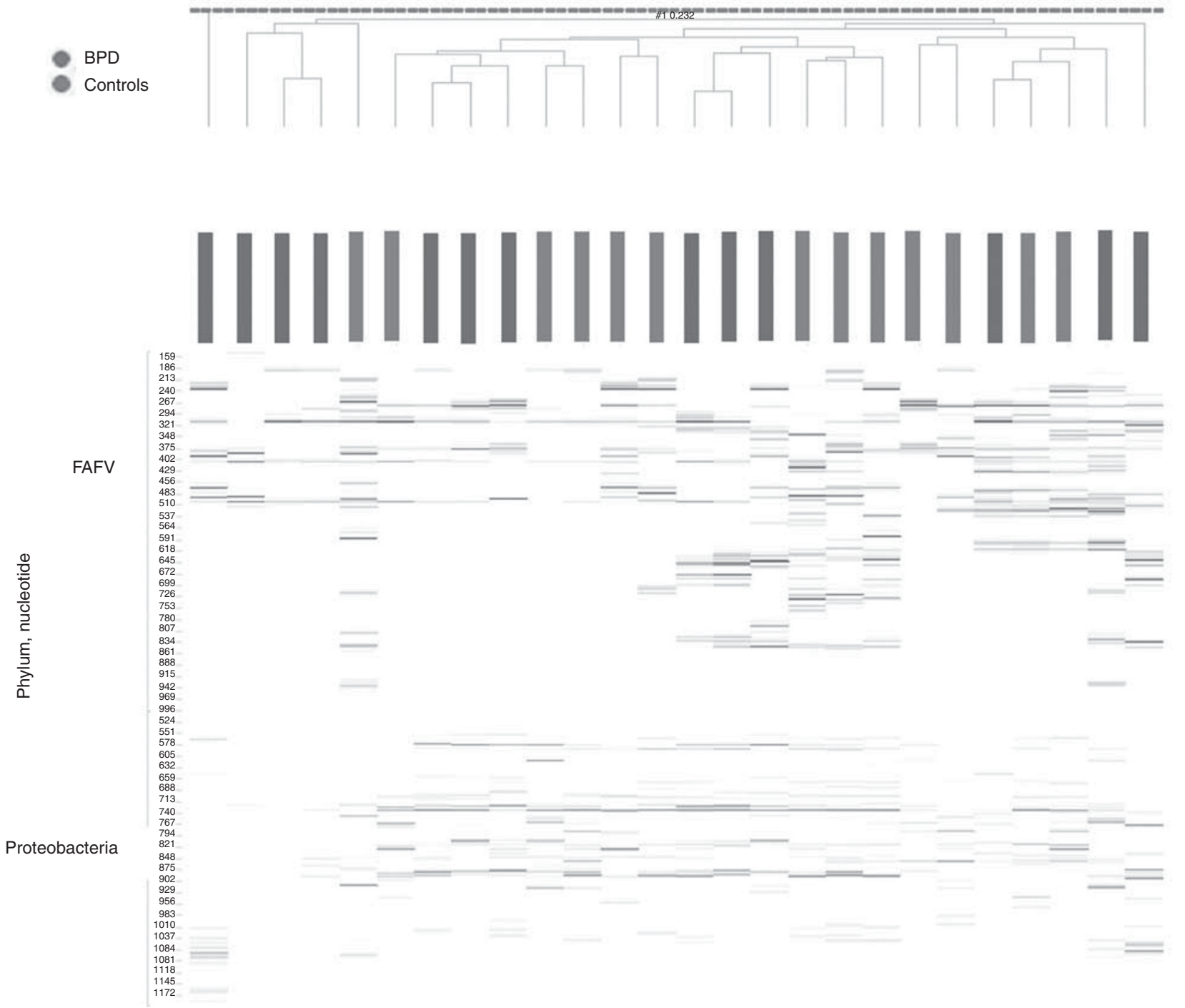

Figure 4. Clustered heatmap displaying IS profiles of 13 infants with severe bronchopulmonary dysplasia (BPD) and 13 matched controls. Individual subjects are shown on the $x$ axis; children with BPD in green, controls in red. On the $y$ axis, IS fragment lengths are expressed (in the number of nucleotides), corresponding with bacterial strain type (Operational Taxonomic Units). Blue peaks represent Firmicutes, Actinibacteria, Fusibacteria, and Verrucomicrobia (FAFV) and yellow peaks represent Proteobacteria. Notably, Bacteriodetes are absent in all the analyzed samples. Intensity of colors reflect relative dominance of each indicated bacterial strain; gray signals represent less prevalent IS fragment lengths. A full color version of this figure is available at the Pediatric Research journal online.

exhaled VOCs may even provide a more integral view on the potential of VOC profiling as an early diagnostic biomarker for BPD and may increase understanding of the pathophysiological metabolic processes involved in development of (severe) BPD.

Strengths of this study are the multicenter design, robust collection of both clinical and demographic data, and strict matching procedure allowing for adequate group comparison. This study also has important limitations. First, we have not been able to compare fecal VOC profiles with those in exhaled breath. Second, a relatively small sample size was analyzed owing to stringent matching criteria and exclusion of mild and moderate BPD cases. However, by including only severe BPD cases, we created a homogeneous study group with only limited interindividual variability, as it has become evident that severe BPD may be a different entity from mild and moderate BPD, both in terms of clinical definition as well as in terms of genetic predisposition (40). Third, to determine the required fraction of inspired oxygen $\left(\mathrm{FiO}_{2}\right)$ and consequently the severity of $\mathrm{BPD}$, an oxygen reduction test at the PMA of 36 weeks should be performed. However, as this test was not routinely performed in all referral centers, we used the administered oxygen percentage at 36 weeks PMA to determine BPD severity. Another limitation is that BPD infants with a history of necrotizing enterocolitis and/or pneumonia were excluded, although these infants have an increased risk for developing BPD. We did so to minimize the risk of false-positive VOC profile outcomes; infants with necrotizing enterocolitis are characterized by a significantly distinct fecal VOC profile compared with controls (18). In 


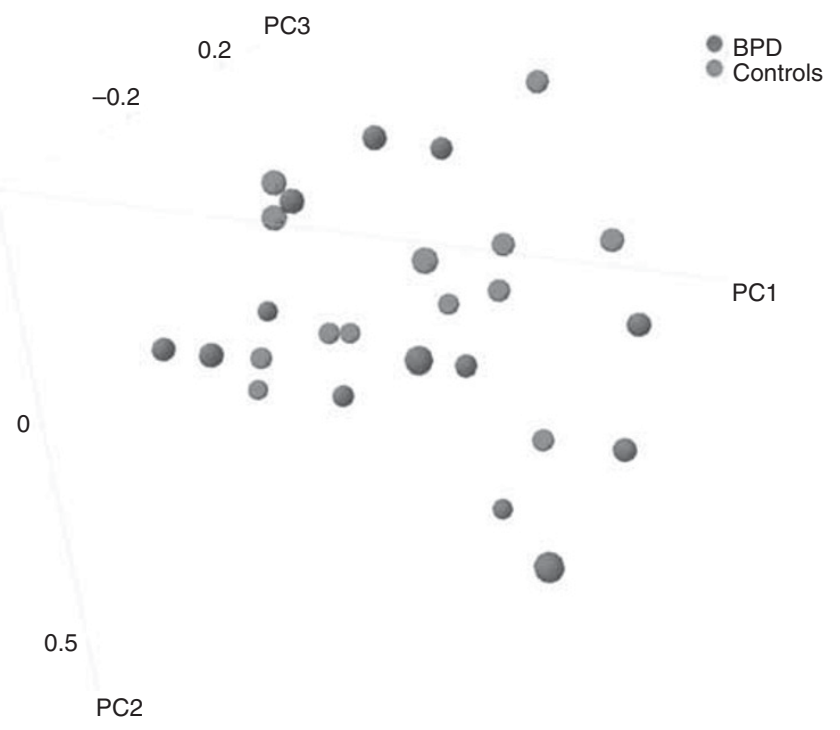

Figure 5. Principal coordinate analysis scatterplot displaying overall bacterial community composition, showing no separate clustering of microbial profiles of children with severe bronchopulmonary dysplasia (BPD; blue dots) and controls (red dots) for all phyla together. A full color version of this figure is available at the Pediatric Research journal online.

addition, long-term administration of antibiotics causes shifts in intestinal microbiota composition and consequently in fecal VOCs. Finally, microbiota analysis was performed at only one time point only. However, we believe that, if an altered intestinal microbiota caused the observed differences in fecal VOCs, this difference in intestinal microbiota would be detectable at the postnatal age of 28 days.

In conclusion, we observed that development of severe BPD was associated with alterations in fecal VOC profiles at postnatal age of 14,21, and 28 days. These differences could not be attributed to intestinal microbiota alterations but presumably reflect local and/or systemic metabolic and inflammatory pathways involved in BPD development. Identification of BPD-specific volatiles may increase understanding of BPD pathophysiology while simultaneous analysis of fecal and exhaled VOCs may provide an integral view on the potential of VOC profiling as an early diagnostic biomarker for BPD.

\section{SUPPLEMENTARY MATERIAL}

Supplementary material is linked to the online version of the paper at http://www.nature.com/pr

\section{ACKNOWLEDGMENTS}

We received an unrestricted grant from Landelijke Vereniging van Crematoria (Dr. C.J. Vaillant Fonds) and Christine Bader Stichting Irene Kinderziekenhuis to conduct this study.

Disclosure: The authors declare no conflict of interest.

\section{REFERENCES}

1. Horbar JD, Carpenter JH, Badger GJ, et al. Mortality and neonatal morbidity among infants 501 to 1500 grams from 2000 to 2009. Pediatrics 2012;129:1019-26.
2. Johnson TJ, Patel AL, Jegier BJ, Engstrom JL, Meier PP. Cost of morbidities in very low birth weight infants. J Pediatr 2013;162: 243-49 e1.

3. Short EJ, Klein NK, Lewis BA, et al. Cognitive and academic consequences of bronchopulmonary dysplasia and very low birth weight: 8-year-old outcomes. Pediatrics 2003;112:e359.

4. Balinotti JE, Chakr VC, Tiller C, et al. Growth of lung parenchyma in infants and toddlers with chronic lung disease of infancy. Am J Respir Crit Care Med 2010;181:1093-7.

5. Vardar-Yagli N, Inal-Ince D, Saglam $M$, et al. Pulmonary and extrapulmonary features in bronchopulmonary dysplasia: a comparison with healthy children. J Phys Ther Sci 2015;27:1761-5.

6. Vom Hove M, Prenzel F, Uhlig HH, Robel-Tillig E. Pulmonary outcome in former preterm, very low birth weight children with bronchopulmonary dysplasia: a case-control follow-up at school age. J Pediatr 2014;164: 40-5 e4.

7. Doyle LW, Halliday HL, Ehrenkranz RA, Davis PG, Sinclair JC. An update on the impact of postnatal systemic corticosteroids on mortality and cerebral palsy in preterm infants: effect modification by risk of bronchopulmonary dysplasia. J Pediatr 2014;165:1258-60.

8. Buijck M, Berkhout DJ, de Groot EF, et al. Sniffing out paediatric gastrointestinal diseases: the potential of volatile organic compounds as biomarkers for disease. J Pediatr Gastroenterol Nutr 2016;63:585-91.

9. Alkhouri N, Eng K, Cikach F, et al. Breathprints of childhood obesity: changes in volatile organic compounds in obese children compared with lean controls. Pediatr Obes 2015;10:23-9.

10. de Meij TG, Larbi IB, van der Schee MP, et al. Electronic nose can discriminate colorectal carcinoma and advanced adenomas by fecal volatile biomarker analysis: proof of principle study. Int J Cancer 2014;134:1132-8.

11. Bomers MK, Menke FP, Savage RS, et al. Rapid, accurate, and on-site detection of C. difficile in stool samples. Am J Gastroenterol 2015;110:588-94.

12. deMeij TG, de Boer NK, Benninga MA, et al. Faecal gas analysis by electronic nose as novel, non-invasive method for assessment of active and quiescent paediatric inflammatory bowel disease: proof of principle study. J Crohns Colitis 2014; pii: S1873-9946(14)00285-2.

13. Budden KF, Gellatly SL, Wood DL, et al. Emerging pathogenic links between microbiota and the gut-lung axis. Nat Rev Microbiol 2017;15:55-63.

14. Schroeder BO, Backhed F. Signals from the gut microbiota to distant organs in physiology and disease. Nat Med 2016;22:1079-89.

15. Jobe AH, Bancalari E. Bronchopulmonary dysplasia. Am J Respir Crit Care Med 2001;163:1723-9.

16. Berkhout DJ, Benninga MA, van Stein RM, et al. Effects of sampling conditions and environmental factors on fecal volatile organic compound analysis by an electronic nose device. Sensors(Basel) 2016;16:E1967.

17. Berkhout DJ, Niemarkt HJ, Buijck M, et al. Detection of sepsis in preterm infants by fecal volatile organic compounds analysis: a proof of principle study. J Pediatr Gastroenterol Nutr 2016;65:e47-52.

18. de Meij TG, van der Schee MP, Berkhout DJ, et al. Early detection of necrotizing enterocolitis by fecal volatile organic compounds analysis. J Pediatr 2015;167:562-7 e1.

19. Li J. The cyranose chemical vapor analyzer. Sensors 2000;17:56-61.

20. Budding AE, Grasman ME, Lin F, et al. IS-pro: high-throughput molecular fingerprinting of the intestinal microbiota. FASEB J 2010;24: 4556-64.

21. de Meij TG, Budding AE, de Groot EF, et al. Composition and stability of intestinal microbiota of healthy children within a Dutch population. FASEB J 2016;30:1512-22.

22. Rogosch T, Herrmann N, Maier RF, et al. Detection of bloodstream infections and prediction of bronchopulmonary dysplasia in preterm neonates with an electronic nose. J Pediatr 2014;165:622-4.

23. Sever A, Abd Elkadir A, Matana Y, Gopas J, Zeiri Y. Biomarkers for detection and monitoring of B16 melanoma in mouse urine and feces. J Biomark 2015;2015:841245.

24. Lohmann P, Luna RA, Hollister EB, et al. The airway microbiome of intubated premature infants: characteristics and changes that predict the 
development of bronchopulmonary dysplasia. Pediatr Res 2014;76: 294-301.

25. van der Schee MP, Paff T, Brinkman P, van Aalderen WM, Haarman EG, Sterk PJ. Breathomics in lung disease. Chest 2015;147:224-31.

26. May C, Patel S, Kennedy C, et al. Prediction of bronchopulmonary dysplasia. Arch Dis Child Fetal Neonatal Ed 2011;96:F410-6.

27. May C, Williams O, Milner AD, et al. Relation of exhaled nitric oxide levels to development of bronchopulmonary dysplasia. Arch Dis Child Fetal Neonatal Ed 2009;94:F205-9.

28. Imamura $\mathrm{T}$, Sato $\mathrm{M}$, Go $\mathrm{H}$, et al. The microbiome of the lower respiratory tract in premature infants with and without severe bronchopulmonary dysplasia. Am J Perinatol 2017;34:80-7.

29. Stressmann FA, Connett GJ, Goss K, et al. The use of culture-independent tools to characterize bacteria in endo-tracheal aspirates from pre-term infants at risk of bronchopulmonary dysplasia. J Perinat Med 2010;38:333-7.

30. Marsland BJ, Trompette A, Gollwitzer ES. The gut-lung axis in respiratory disease. Ann Am Thorac Soc 2015;12 (Suppl 2): S150-6.

31. Trompette A, Gollwitzer ES, Yadava K, et al. Gut microbiota metabolism of dietary fiber influences allergic airway disease and hematopoiesis. Nat Med 2014;20:159-66.

32. Chen LW, Chen PH, Hsu CM. Commensal microflora contribute to host defense against Escherichia coli pneumonia through Toll-like receptors. Shock 2011;36:67-75.
33. Yoon BH, Romero R, Kim KS, et al. A systemic fetal inflammatory response and the development of bronchopulmonary dysplasia. Am J Obstet Gynecol 1999;181:773-9.

34. Choi CW, Lee J, Oh JY, Lee SH, Lee HJ, Kim BI. Protective effect of chorioamnionitis on the development of bronchopulmonary dysplasia triggered by postnatal systemic inflammation in neonatal rats. Pediatr Res 2016;79:287-94.

35. Saugstad OD. Oxygen and oxidative stress in bronchopulmonary dysplasia. J Perinat Med 2010;38:571-7.

36. Balena-Borneman J, Ambalavanan N, Tiwari HK, Griffin RL, Halloran B, Askenazi D. Biomarkers associated with bronchopulmonary dysplasia/ mortality in premature infants. Pediatr Res 2016;81:519-25.

37. Rivera L, Siddaiah R, Oji-Mmuo C, Silveyra GR, Silveyra P. Biomarkers for bronchopulmonary dysplasia in the preterm infant. Front Pediatr 2016;4:33.

38. Doyle LW, Ehrenkranz RA, Halliday HL. Early ( $<8$ days) postnatal corticosteroids for preventing chronic lung disease in preterm infants. Cochrane Database Syst Rev 2014 (5) CD001146.

39. Lal CV, Ambalavanan N. biomarkersearly diagnosis, and clinical predictors of bronchopulmonary dysplasia. Clin Perinatol 2015;42: 739-54.

40. Lal CV, Ambalavanan N. Genetic predisposition to bronchopulmonary dysplasia. Semin Perinatol 2015;39:584-91. 